TITLE: Citrullinated Chemokines in Rheumatoid Arthritis

PRINCIPAL INVESTIGATOR: $\quad$ David A. Fox

CONTRACTING ORGANIZATION: Regents of the University of Michigan

Ann Arbor, MI 48109-1274

REPORT DATE: $\quad$ October 2014

TYPE OF REPORT: Annual

PREPARED FOR: $\quad$ U.S. Army Medical Research and Materiel Command Fort Detrick, Maryland 21702-5012

DISTRIBUTION STATEMENT: $\quad$ Approved for Public Release;

Distribution Unlimited

The views, opinions and/or findings contained in this report are those of the author(s) and should not be construed as an official Department of the Army position, policy or decision unless so designated by other documentation. 


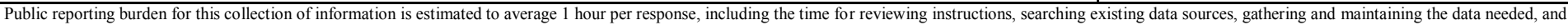

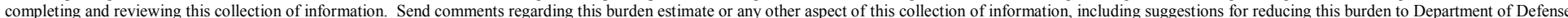

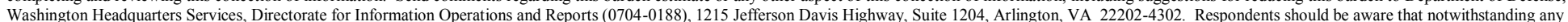

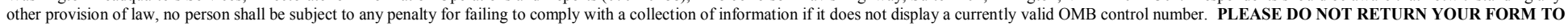
THE ABOVE ADDRESS.

\begin{tabular}{|l|l} 
1. REPORT DATE O ctober 2014 & 2. REPORT TYPE Annual
\end{tabular}

4. TITLE AND SUBTITLE

Citrullinated Chemokines in Rheumatoid Arthritis

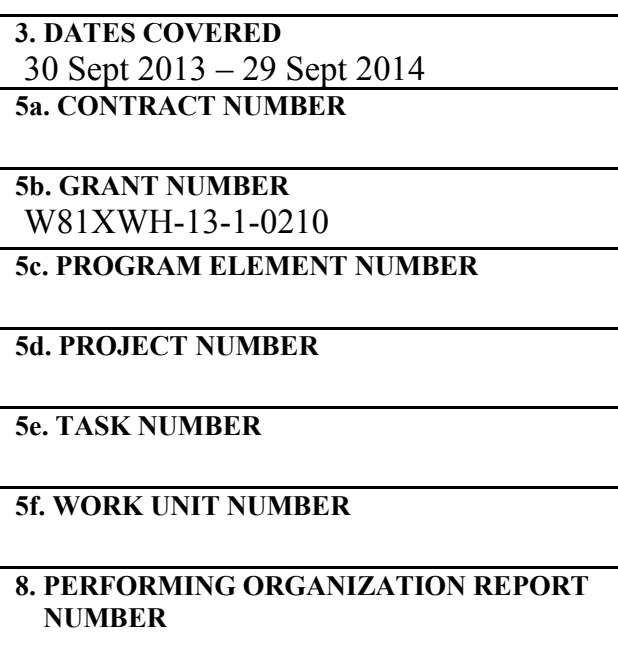

\section{AUTHOR(S)}

David A. Fox, MD

E-Mail: dfox@umich.edu

7. PERFORMING ORGANIZATION NAME(S) AND ADDRESS(ES)

Regents of The University Of Michigan

Ann Arbor, MI 48109-1340

9. SPONSORING / MONITORING AGENCY NAME(S) AND ADDRESS(ES)

10. SPONSOR/MONITOR'S ACRONYM(S)

U.S. Army Medical Research and Materiel Command

Fort Detrick, Maryland 21702-5012

11. SPONSOR/MONITOR'S REPORT

NUMBER(S)

\section{DISTRIBUTION / AVAILABILITY STATEMENT}

Approved for Public Release; Distribution Unlimited

\section{SUPPLEMENTARY NOTES}

\section{ABSTRACT}

Citrullination, catalysed by peptidylarginine deiminase (PAD), is a post-translational modification of arginine to citrulline, which contributes to the pathogenesis of rheumatoid arthritis (RA). We show that citrullinated epithelialderived neutrophil-activating peptide 78/CXCL5 (cit-ENA-78/CXCL5) is significantly higher in RA synovial fluids (SFs) compared to osteoarthritis (OA) and other inflammatory rheumatic diseases (OD) SFs, and its concentration correlates with RA disease activity. Citrullinated chemokine concentrations were measured by enzyme linked immunosorbent assay (ELISA) in RA and normal (NL) sera and in RA, osteoarthritis (OA), and other inflammatory rheumatic disease (OD) synovial fluids (SFs). The correlation between the citrullinated chemokine levels and clinical data was analyzed. A strong correlation was found between the amount of citrullinated ENA-78/CXCL5 and C-reactive protein or erythrocyte sedimentation rate (ESR) in RA SFs. These results indicate that citrullinated ENA78/CXCL5 may have novel inflammatory properties in RA pathogenesis.

\section{SUBJECT TERMS}

Citrullination, chemokines, chemotaxis, rheumatoid arthritis, immunology

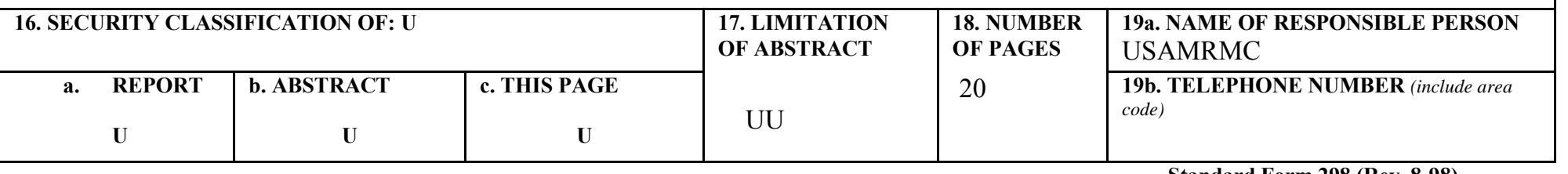




\section{Table of Contents}

$\underline{\text { Page }}$

1. Introduction......................................................... 4

2. Keywords...................................................... 4

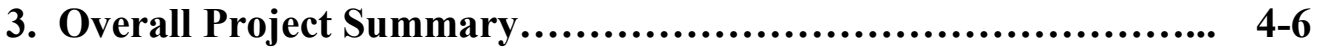

4. Key Research Accomplishments................................. 6

5. Conclusion....................................................... 6

6. Publications, Abstracts, and Presentations..................... 6-7

7. Inventions, Patents and Licenses............................... 7

8. Reportable Outcomes............................................... 7

9. Other Achievements............................................... 7

10. References.................................................... 7

11. Appendices.................................................... 8-21 


\section{Introduction}

Citrullination, catalysed by PAD enzymes, is a post-translational modification of arginine to citrulline that contributes to the pathogenesis of rheumatoid arthritis (RA). Chemokines, which play an important role in the development of RA, can be citrullinated in vitro. We are examining whether citrullinated chemokines are detected in RA biological fluids, and if so, what their biological activities are. We posit that modification of seminal chemokines in the RA SF confers unique properties that significantly modify the inflammatory environment of the RA joint. Chemokines, including epithelial-derived neutrophilactivating peptide 78 (ENA-78/CXCL5), play a critical role in the development of RA, and are involved in inflammatory leukocyte migration into rheumatoid synovial tissue. Recent studies have shown that several chemokines, ENA-78/CXCL5, interleukin-8 (IL-8/CXCL8), interferon gamma-induced protein 10 (IP-10/CXCL10), interferon-inducible T-cell alpha chemoattractant (I-TAC/CXCL11), and stromal derived factor-1 $\alpha$ (SDF-1 $\alpha /$ CXCL12), can be citrullinated by peptidyl arginine deiminase (PAD) in vitro. In this study, we want to investigate whether ENA-78/CXCL5, macrophage inflammatory protein-1 $\alpha$ (MIP-1 $\alpha /$ CCL3), and monocyte chemotactic protein-1 (MCP-1/CCL2), which are representative chemokines in RA, are citrullinated in RA, osteoarthritis (OA), and normal (NL) control subjects. We then want to examine the properties of these modified chemokines to explore their novel functions that will offer additional insight into the function of chemokines in chronic diseases such as RA.

\section{Keywords}

Rheumatoid arthritis (RA)

Osteoarthritis (OA)

Normal (NL)

Synovial Fluid (SF)

Synovial Tissue (ST)

Serum

Chemokines

Chemotaxis

Anti-cyclic-citrullinated peptide antibodies (anti-CCP or ACPA)

Liquid chromatography coupled with tandem mass spectrometry (LC-MS/MS)

Citrullination

Enzyme Linked Immunosorbant Assay (ELISA)

Epithelial Neutrophil Chemoattractant Peptide-78 (ENA-78/CXCL5)

Monocyte Chemoattractant Protein-1 (MCP-1/CCL2)

Macrophage Inflammatory Protein-1 alpha (MIP-1 $\alpha /$ CCL3)

Peptidyl Arginine Deiminase (PAD)

Neutrophils (PMNs)

Monocytes

Arginine

Post-translational

\section{Overall Project Summary}

Current Objectives: Specific Aim 1: To determine how citrullinated chemokines compare in RA, vs. OA, vs. other rheumatic diseases (OD), vs. NLs.

Major Task 1: Characterize citrullinated chemokines in RA.

Subtask 1: Synovial fluids (SF) and sera will be obtained from patients with RA, OA or ODs and assayed for key citrullinated and non-citrullinated chemokines thought to be important in the pathogenesis of RA. 
Subtask 2: Synovial tissue (ST) will be obtained from RA, OA, or NL joints, homogenized and assayed for citrullinated and non-citrullinated chemokines.

Summary of Results: We have been actively recruiting patients for this study. We currently have enough patient fluids and tissues to perform all assays proposed in this grant. We have generated data to show that ENA-78/CXCL5, MIP-1 $\alpha /$ CCL3 and MCP-1/CCL2 can all be detected in RA SFs. We have made tissue homogenates obtained from patients with RA and OA, and from NL ST obtained from tissue repositories. Using these homogenates, we have shown that RA ST joint homogenates contain citrullinated ENA-78/CXCL5 and that this can be detected by Western blotting analysis.

Progress and Accomplishments: Citrullination is a post-translational modification mediated by PAD. Chemokines, which play an important role in the development of RA, can be citrullinated in vitro. We undertook this study to examine whether the citrullinated chemokines ENA-78/CXCL5, MIP-1 $\alpha /$ CCL3, and MCP-1/CCL2 are detected in RA biological fluids, and if so, what their biological activities are. Recombinant human chemokines were citrullinated by PAD. Citrullinated chemokine concentrations were measured by enzyme linked immunosorbent assay in RA and normal sera and in RA, OA, and OD SFs. The correlation between the citrullinated chemokine levels and clinical data was analyzed. We found that citrullinated ENA-78/CXCL5 was significantly higher in RA sera and SFs than normal sera and OD including OA SFs, respectively (figure 1). A strong correlation was found between the amount of citrullinated ENA-78/CXCL5 and C-reactive protein or erythrocyte sedimentation rate in RA SFs. Our conclusions for the first year of experiments is that citrullinated ENA-78/CXCL5 can be detected in RA tissues and is highly correlated with RA disease activity.

Taken together, evidence is strong for citrullination being integral to inflammation and the pathogenesis of RA. Inflammatory cytokines are known to be perpetuators of the disease process, but they may also be more than that. If our hypothesis is correct, citrullinated chemokines may be present and pathogenic early in the course of RA. These preliminary studies should establish whether this is the case

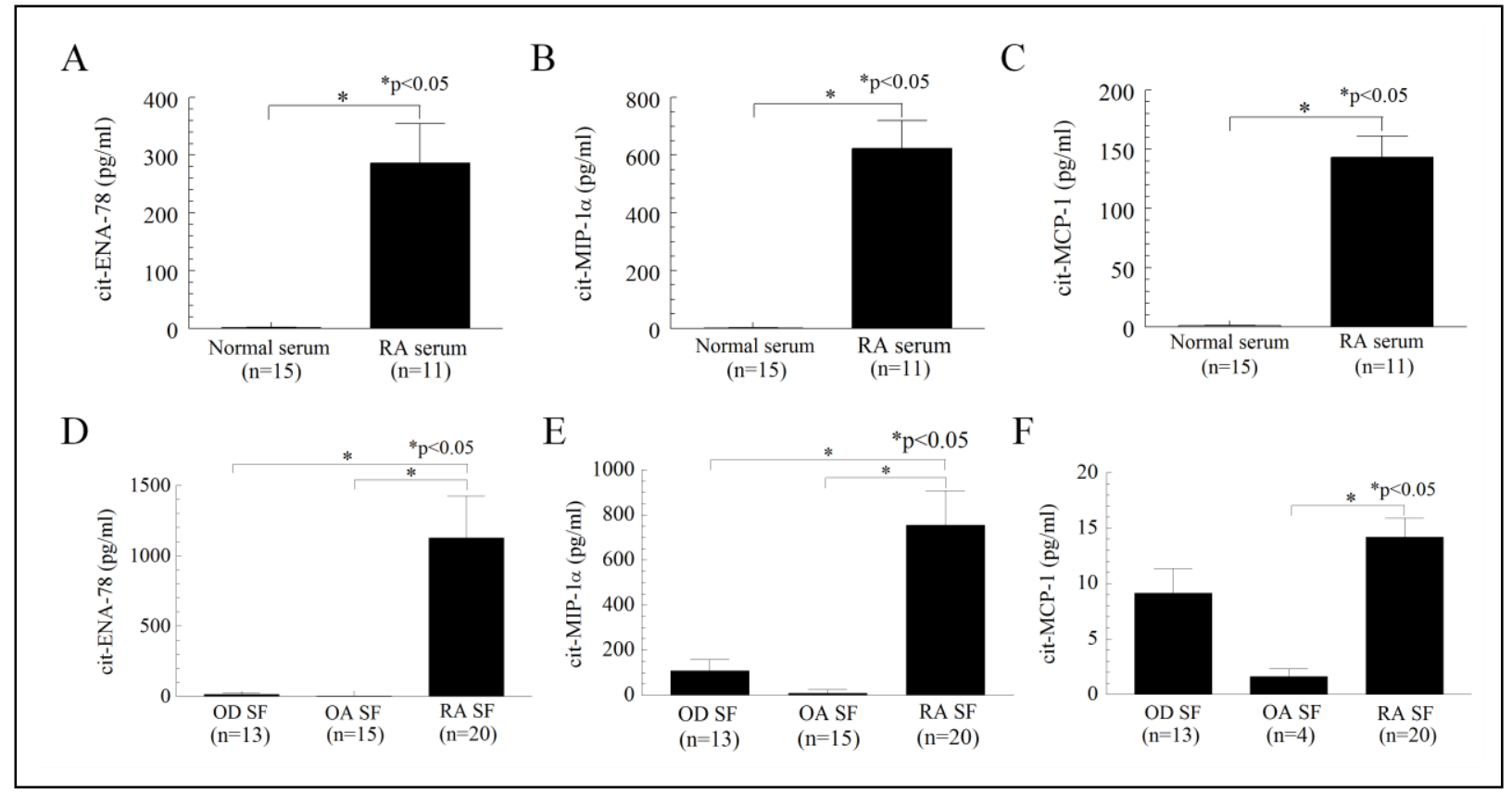

Figure 1.Citrullinated chemokines are highly expressed in RA sera and SFs. Citrullinated chemokines were measured using an ELISA in which chemokines were captured on an ELISA plate followed by detection with anti-modified citrulline after chemical modification of citrulline residues. RF (rheumatoid factor) was depleted from RA SFs and sera prior to the ELISA assay. A), B), and C), Citrullinated ENA-78/CXCL5, MIP-1 $\alpha / \mathrm{CCL} 3$, and MCP-1/CCL2 concentrations in RA and normal sera. These citrullinated chemokines were all greater in RA compared to normal sera. D), E), and F) Citrullinated ENA-78/CXCL5, MIP-1 $\alpha /$ CCL3, and MCP-1/CCL2 concentrations in RA, OD, and OA SFs. Citrullinated ENA-78/CXCL5 and MIP-1 $\alpha /$ CCL3 were significantly greater in RA SFs than in OA and OD SFs. Citrullinated MCP-1/CCL2 was higher in RA compared to OA SFs. Bars show the mean $\pm \mathrm{SEM}\left(\mathrm{n}=\right.$ the number of patients). ${ }^{*} \mathrm{p}<0.05$ was considered significant. cit-ENA$78=$ citrullinated ENA-78/CXCL5; cit-MIP-1 $\alpha=$ citrullinated MIP-1 $\alpha /$ CCL3; cit-MCP-1 = citrullinated MCP-1/CCL2. 
for key chemokines involved in RA pathogenesis. The results of these studies will also help us determine if citrullinated chemokines should be pursued as potential biomarkers for RA. We have recently published a manuscript supporting our hypothesis ${ }^{1}$, and have shown that citrullination can effect chemokine activity and be associated with markers of disease severity.

\section{References:}

1. Yoshida Y, Korchynskyi O, Tak PP, Isozaki T, Ruth JH, Campbell PL, Baeten DL, Gerlag DM, Amin MA, Koch AE: Citrullination of ENA-78/CXCL5 results in conversion from a non-monocyte recruiting to a monocyte recruiting chemokine. Arthritis Rheumatol. In press.

\section{Key Research Accomplishments}

- Citrullinated ENA-78/CXCL5 was significantly higher in RA sera and SFs than NL sera and OD including OA SFs, respectively.

- A strong correlation was found between the amount of citrullinated ENA-78/CXCL5 and Creactive protein or erythrocyte sedimentation rate (ESR) in RA SFs.

- Our conclusions for the first year of experiments is that citrullinated ENA-78/CXCL5 can be detected in RA tissues and is highly correlated with RA disease activity.

- We have generated mutants for our project (as proposed) and now have constructs of ENA78/CXCL5 that are non-mutated, and two others that have the arginine replaced by a lysine residue (Origene). These mutant forms will be tested against non-mutated ENA-78/CXCL5 to examine its cell recruitment activity in citrullinated and non-citrullinated forms.

- Mice have been immunized with citrullinated ENA-78/CXCL5 to produce a monoclonal antibody to the modified form of this pro-inflammatory chemokine. If successful in isolating the B-Cell clone, we will isolate and use these antibodies to examine the expression and function of citrullinated ENA-78/CXCL5 in RA tissues.

\section{Conclusion}

Citrullinated chemokine concentrations were measured by enzyme linked immunosorbent assay in RA and NL sera and in RA, OA, and OD SFs. The correlation between the citrullinated chemokine levels and clinical data was analyzed. We found that citrullinated ENA-78/CXCL5 was significantly higher in RA sera and SFs than NL sera and OD including OA SFs, respectively. A strong correlation was found between the amount of citrullinated ENA-78/CXCL5 and C-reactive protein or erythrocyte sedimentation rate in RA SFs. Our conclusions for the first year of experiments is that citrullinated ENA-78/CXCL5 can be detected in RA tissues and is highly correlated with RA disease activity. Taken together, evidence is strong for citrullination being integral to inflammation and the pathogenesis of RA. Inflammatory cytokines are known to be perpetuators of the disease process. If our hypothesis is correct, citrullinated chemokines may be present and pathogenic early in the course of RA and may be key biological markers that could be detected before disease onset.

\section{Publications, Abstracts and Presentations}

\section{Peer-reviewed manuscripts:}


1. *Yoshida Y, Korchynskyi O, Tak PP, Isozaki T, Ruth JH, Campbell PL, Baeten DL, Gerlag DM, Amin MA, Koch AE: Citrullination of ENA-78/CXCL5 results in conversion from a nonmonocyte recruiting to a monocyte recruiting chemokine. Arthritis Rheumatol. In press.

Abstracts presented at national meetings:

1. *Yoshida K, Campbell PL, Amin MA, Koch AE, Fox DA, Ruth JH: Citrullination of ENA78/CXCL5 changes its receptor affinity from CXCR2 to CXCR1 and induces monocyte migration. Arthritis Rheum. 65 S10:327, 2013.

2. Edhayan G, Ha CM, Ohara RA, Isozaki T, Amin MA, Arbab AS, Tsou PS, Campbell PL, Schiopu E, Khanna D, Morgan R, Friday SC, Fox DA, Ruth JH: Inflammatory Properties of Inhibitor of DNA Binding 1 as a Unique Fibroblast Derived Nuclear Protein. Arthritis Rheumatol. In press.

3. Ohara RA, Edhayan G, Ha CM, Amin MA, Arbab AS, Campbell PL, Fox DA, Ruth JH: Evidence for citrullination of the nuclear transcription factor Inhibitor of DNA Binding 1 (Id1) in Rheumatoid Arthritis. Arthritis Rheumatol. In press.

\section{Inventions, Patents and Licenses}

Nothing to report.

\section{Reportable outcomes}

- We developed an ELISA that can successfully detect citrullinated chemokines in RA tissues.

- We developed a Western blotting technique that can successfully detect citrullinated chemokines in RA tissues.

- We can correlate RA disease severity with citrullinated ENA-78/CXCL5 concentrations in RA SF.

\section{Other Achievements}

Nothing to report.

\section{References}

1. Yoshida Y, Korchynskyi O, Tak PP, Isozaki T, Ruth JH, Campbell PL, Baeten DL, Gerlag DM, Amin MA, Koch AE: Citrullination of ENA-78/CXCL5 results in conversion from a nonmonocyte recruiting to a monocyte recruiting chemokine. Arthritis Rheumatol. In press.

\section{Appendices}

Please see manuscript (reference 1) enclosed. 


\section{Quad/Gantt chart}

\begin{tabular}{|c|c|c|c|}
\hline \multicolumn{4}{|c|}{ TIMETABLE FOR RESEARCH PROGRAM } \\
\hline & YEAR 01 & YEAR 02 & YEAR 03 \\
\hline $\begin{array}{c}\text { SPECIFIC } \\
\text { AIM \#1 }\end{array}$ & $\begin{array}{c}\text { COMPLETED: } \\
\text { Synovial fluids and sera will be } \\
\text { obtained from patients with RA, } \\
\text { OA or other diseases and assayed } \\
\text { for key citrullinated and non- } \\
\text { citrullinated chemokines thought to } \\
\text { be important in the pathogenesis of } \\
\text { RA. }\end{array}$ & $\begin{array}{l}\text { COMPLETED: Synovial } \\
\text { tissue will be obtained from } \\
\text { RA, OA, or NL joints, } \\
\text { homogenized and assayed for } \\
\text { citrullinated and non- } \\
\text { citrullinated chemokines. }\end{array}$ & \\
\hline $\begin{array}{l}\text { SPECIFIC } \\
\text { AIM \#2 }\end{array}$ & $\begin{array}{l}\text { 75\% COMPLETE: Leukocyte } \\
\text { chemotaxis assays, modifying } \\
\text { chemokines containing ELR motif } \\
\text { and mass spec analysis, site } \\
\text { directed mutagenesis of } \\
\text { chemokines. }\end{array}$ & $\begin{array}{c}\text { 25\% COMPLETE: Signal } \\
\text { transduction analysis of } \\
\text { endothelial cells and } \\
\text { leukocytes. }\end{array}$ & $\begin{array}{l}\text { In vitro Angiogenesis assays } \\
\text { and Matrigel plug assay (if } \\
\text { needed) }\end{array}$ \\
\hline $\begin{array}{c}\text { SPECIFIC } \\
\text { AIM \#3 }\end{array}$ & & $\begin{array}{l}50 \% \text { COMPLETE: Sera } \\
\text { obtained from RA, OA, or } \\
\text { NL individuals will be } \\
\text { assayed against citrullinated } \\
\text { vs. non-citrullinated } \\
\text { chemokines to determine if } \\
\text { autoantibodies are made. }\end{array}$ & $\begin{array}{l}\text { Citrullinated chemokines will } \\
\text { be adsorbed out of RA serum } \\
\text { to determine how much } \\
\text { reactivity for citrullinated } \\
\text { antigens remains. }\end{array}$ \\
\hline $\begin{array}{c}\text { SPECIFIC } \\
\text { AIM \#4 }\end{array}$ & & $\begin{array}{l}\text { Determine the inflammatory } \\
\text { effect of injecting } \\
\text { citrullinated chemokines vs. } \\
\text { native chemokines into } \\
\text { normal rat knees. }\end{array}$ & $\begin{array}{l}\text { Perform rat adjuvant induced } \\
\text { arthritis and determine whether } \\
\text { citrullinated chemokines and } \\
\text { antibodies to these chemokines } \\
\text { are present before and during } \\
\text { development of arthritis. }\end{array}$ \\
\hline
\end{tabular}




\title{
Citrullination of Epithelial Neutrophil-Activating Peptide 78/CXCL5 Results in Conversion From a Non-Monocyte-Recruiting Chemokine to a Monocyte-Recruiting Chemokine
}

\author{
Ken Yoshida, ${ }^{1}$ Olexandr Korchynskyi, ${ }^{2}$ Paul P. Tak, ${ }^{2}$ Takeo Isozaki, ${ }^{1}$ Jeffrey H. Ruth, ${ }^{1}$ \\ Phillip L. Campbell, ${ }^{1}$ Dominique L. Baeten, ${ }^{2}$ Danielle M. Gerlag, ${ }^{2}$ \\ M. Asif Amin, ${ }^{1}$ and Alisa E. Koch ${ }^{3}$
}

Objective. To examine whether the citrullinated chemokines epithelial neutrophil-activating peptide 78 (ENA-78)/CXCL5, macrophage inflammatory protein $1 \alpha / C C L 3$, and monocyte chemotactic protein $1 /$ CCL2 are detected in the biologic fluid of patients with rheumatoid arthritis (RA), and if so, to determine the biologic activities of these chemokines.

Methods. Recombinant human chemokines were citrullinated by peptidylarginine deiminase. Enzymelinked immunosorbent assays were performed to measure the concentrations of citrullinated chemokines in sera from patients with rheumatoid arthritis (RA) and normal individuals and in synovial fluid from patients with RA, patients with osteoarthritis (OA), and patients with other inflammatory rheumatic diseases. The correlation between the citrullinated chemokine levels and clinical data was analyzed. Monocyte and neutrophil chemotaxis assays were performed, and native (non-

Supported by the Office of Research and Development, Medical Research Service, Department of Veterans Affairs and by the Frederick G. L. Huetwell and William D. Robinson, MD, Professorship in Rheumatology at the University of Michigan (awarded to Dr. Koch).

${ }^{1}$ Ken Yoshida, MD, PhD, Takeo Isozaki, MD, PhD, Jeffrey H. Ruth, PhD, Phillip L. Campbell, BA, M. Asif Amin, MD: University of Michigan, Ann Arbor; ${ }^{2}$ Olexandr Korchynskyi, PhD, Paul P. Tak, MD, PhD, Dominique L. Baeten, MD, PhD, Danielle M. Gerlag, MD, PhD: University of Amsterdam, Amsterdam, The Netherlands; ${ }^{3}$ Alisa E. Koch, MD: Department of Veterans Affairs Medical Center, Ann Arbor, and University of Michigan, Ann Arbor.

Drs. Yoshida and Korchynskyi contributed equally to this work.

Address correspondence to Alisa E. Koch, MD, University of Michigan Medical School, Ann Arbor, MI 48109-2200. E-mail: aekoch@umich.edu.

form June 12, 2014. citrullinated) or citrullinated ENA-78/CXCL5 was injected into mouse knees to evaluate the biologic activities of these chemokines.

Results. The concentration of citrullinated ENA78/CXCL5 was significantly higher in RA sera and SF than in normal sera and in SF from patients with other rheumatic diseases including OA. In RA SF, a strong correlation between the amount of citrullinated ENA78/CXCL5 and the C-reactive protein level or the erythrocyte sedimentation rate was observed. Citrullinated ENA-78/CXCL5 induced monocyte chemotaxis via CXCR1 and CXCR2, while noncitrullinated ENA-78/ CXCL5 did not. In a mouse model of inflammatory arthritis, citrullinated ENA-78/CXCL5 induced more severe inflammation and recruited more monocytes than did noncitrullinated ENA-78/CXCL5.

Conclusion. Citrullinated ENA-78/CXCL5 is highly correlated with RA disease activity and, unlike noncitrullinated ENA-78/CXCL5, recruits monocytes. These results indicate that citrullinated ENA-78/CXCL5 may exert previously unrecognized inflammatory properties in RA by recruiting monocytes to inflamed joint tissue.

Rheumatoid arthritis (RA) is a chronic inflammatory disorder characterized by the infiltration of inflammatory cells into synovial tissue and synovial fluid (SF) (1). Anti-cyclic citrullinated peptide (anti-CCP) antibodies are present in the sera of nearly $70 \%$ of patients with RA and have become key diagnostic markers for this disease (2). Citrulline residues in target antigens are essential in the formation of anticitrullinated peptide/protein antibody epitopes $(3,4)$. These epitopes result from citrullination, a posttransla- 
tional modification of arginine. Peptidylarginine deiminase (PAD) enzymes are calcium-dependent enzymes that catalyze citrullination of target proteins by converting arginine to citrulline (5). Five PAD family members, PAD type 1 (PAD1), PAD2, PAD3, PAD4, and PAD6, have been identified in many types of tissue, including synovial tissue, and in human leukocytes (6).

Chemokines play an important role as monocyte and polymorphonuclear neutrophil (PMN) recruiters in the setting of RA synovitis and tissue destruction $(7,8)$. Chemokines are classified into 4 subfamilies based on the number and spacing of their first cysteine residues in the primary amino acid sequence (9). These chemokine families are designated as $\mathrm{CXC}, \mathrm{CC}, \mathrm{C}$, and $\mathrm{CX}_{3} \mathrm{C}$. The $\mathrm{CXC}$ chemokines are further divided into 2 subgroups based on whether the Glu-Leu-Arg (ELR) motif precedes the first cysteine residue. Epithelial neutrophilactivating peptide 78 (ENA-78)/CXCL5, a CXC chemokine, is an 8.3-kd protein with 78 amino acids containing 4 cysteine residues positioned identically to those of interleukin-8 (IL-8/CXCL8) (10). We previously observed that the concentration of ENA-78/CXCL5, which is associated with neutrophil recruitment, is significantly higher in RA SF compared with osteoarthritis (OA) SF or SF from patients with other forms of arthritis (11). In addition, we demonstrated that neutralization of ENA78/CXCL5 ameliorated the severity of adjuvant-induced arthritis in a rat model (12). These findings support the notion that ENA-78/CXCL5 is important in the pathogenesis of RA.

Although the presence of citrullinated proteins such as fibrinogen, $\alpha$-enolase, and vimentin in RA has been reported (4,13-15), citrullinated chemokines have not yet been detected in RA. We chose to examine chemokines, because they are biologically relevant joint proteins. Several chemokines are highly expressed in the joints of patients with RA, and PAD2 and PAD4 are mainly present in synovial tissue. The expression levels of these PADs are correlated with inflammation, thickness of the synovial lining layer, and vascularity (16). These observations support the hypothesis that citrullinated chemokines may be present in rheumatic joints.

We compared the presence of citrullinated ENA78/CXCL5, citrullinated macrophage inflammatory protein $1 \alpha(\mathrm{MIP}-1 \alpha) / \mathrm{CCL} 3$, and citrullinated monocyte chemotactic protein 1 (MCP-1)/CCL2 in the biologic fluid of patients with RA with that in the biologic fluid of patients with other arthritic diseases, using a newly developed enzyme-linked immunosorbent assay (ELISA) system, and examined the biologic activity of these chemokines in vitro and that of ENA-78/CXCL5 in vivo.

\section{PATIENTS AND METHODS}

Patients and normal control subjects. Serum samples were obtained from 11 patients with RA and 15 normal control subjects, and SF samples were obtained from 20 patients with RA, 15 patients with OA, and 13 patients with other inflammatory rheumatic diseases, including gout $(n=4)$, pseudogout $(\mathrm{n}=2)$, psoriatic arthritis $(\mathrm{n}=1)$, spondyloarthritis $(\mathrm{n}=3)$, Behçet's disease $(\mathrm{n}=1)$, Lyme disease $(\mathrm{n}=1)$, and unclassified arthritis $(n=1)$. Rheumatoid factor $(R F)$ was immunodepleted from RA sera and SF using goat anti-human IgM ( $\mu$-chain specific) agarose (Sigma-Aldrich) prior to measuring citrullinated chemokines by ELISA. All samples were obtained after approval by the Institutional Review Board and provision of informed consent by the subjects.

Chemokine DNA cloning. Full-length ENA-78/CXCL5 complementary DNA (cDNA) (NCBI accession no. NM_002994) was obtained from Open Biosystems. We generated cDNA libraries for MCP-1/CCL2 and MIP-1 $\alpha /$ CCL3 from primary synovial fibroblasts derived from patients with RA. The cDNA fragments encoding mature proteins were amplified by polymerase chain reaction (PCR) using primers with an incorporated Nco I restriction site in the forward primers and with 6 codons encoding histidines followed by a stop codon and the Eco RI restriction site in the reverse primers (Primer Express software; Applied Biosystems). The primers used are as follows (forward and reverse, respectively): for ENA-78/CXCL5, 5'-TAATCCATGGGAGCTGGTCCTGCCGCTGCTGT-3' and 5'-TAAGAATTCTCAGTGATGGTGATGGTGATGGTTTTCCTTGTTTCCACCGT-3'; for MIP- $1 \alpha /$ CCL3， 5'-TAATCCATGGGAGCTGACACGCCGACCGCCTG-3' and 5'-TAAGAATTCTCAGTGATGGTGATGGTGATGGGCACTCAGCTCCAGGTCGC-3'; for MCP-1/CCL2, 5' -TAATCCATGGGACAGCCAGATGCAATCAATGCC-3' and 5'-TAAGAATTCTCAGTGATGGTGATGGTGATGAGTCTTCGGAGTTTGGGTTTG-3' . Nco $\mathrm{I} / E$ co RI-flanked PCR fragments containing a C-terminus $6 \times$ His tag were initially cloned into a pQE-TriSystem Vector (Qiagen). All sequences were verified using BigDye Terminator sequencing (Life Technologies). For optimization of mammalian expression, inserts containing a C-terminus $6 \times$ His tag were further cloned into the mammalian expression vector pcDEF (17).

Transfection of HEK 293 T cells and purification of recombinant human chemokines. All mammalian expression vectors were transfected into HEK 293T cells using polyethylenimine (Polysciences) to collect $6 \times$ His-tagged chemokines from cellular lysates prepared with $1 \%$ Triton X-100. Chemokines $(6 \times$ His tagged) from HEK $293 \mathrm{~T}$ cell lysates were purified with ProBond Nickel beads (Life Technologies), rinsed extensively with $10 \mathrm{~m} M$ imidazole, and eluted gradually with 50-200 $\mathrm{m} M$ imidazole. The quality and quantity of expressed recombinant proteins were assessed with appropriate DuoSet ELISA kits (R\&D Systems) specific for these proteins and with colloidal Coomassie staining after sodium dodecyl sulfate-polyacrylamide gel electrophoresis resolution.

In vitro citrullination of chemokines. After the concentration of purified chemokines was measured using DuoSet ELISA kits, 100 microliters of purified recombinant human chemokine $(\sim 100 \mathrm{ng} / \mathrm{ml})$ was incubated with 0.5 units of rabbit 
skeletal muscle PAD (Sigma-Aldrich) in $40 \mathrm{~m} M$ Tris $\mathrm{HCl}, \mathrm{pH}$ 7.6, $10 \mathrm{mM} \mathrm{CaCl}$, and $2.5 \mathrm{~m} M$ dithiothreitol for 2 hours at $37^{\circ} \mathrm{C}$. Deimination was stopped by chelating the calcium with $25 \mathrm{~m} M$ EDTA. These citrullinated chemokines were subsequently used as standards for ELISA of citrullinated chemokines. Alternatively, $5 \mu M$ recombinant human ENA-78 (rhENA-78)/CXCL5, recombinant human MIP- $1 \alpha /$ CCL3, or recombinant human MCP-1/CCL2 (OriGene) was incubated with rabbit skeletal PAD $(250 \mathrm{n} M)$ at enzyme:substrate molar ratios of 1:20 in $40 \mathrm{~m} M$ Tris $\mathrm{HCl}$ with $2 \mathrm{mM} \mathrm{CaCl} 2$ (pH 7.4) for 1.5 hours at $37^{\circ} \mathrm{C}$. Deimination was stopped with $0.1 \%$ trifluoroacetic acid (TFA). These citrullinated chemokines were used in both in vitro chemotaxis assays and in vivo experiments. Citrullination of rhENA-78/CXCL5 was confirmed by Western blotting (18). For detection of citrullinated protein, citrulline residues were chemically modified to form a ureido group adduct before addition of an anti-modified citrulline antibody (Millipore) according to the manufacturer's protocol, which enables detection of citrulline residues independent of neighboring amino acid sequences (19). The diagnostic +1Da mass shift occurring upon citrullination was identified by liquid chromatography tandem mass spectrometry (MS Bioworks) (20).

Sandwich ELISA for citrullinated chemokines. An ELISA was designed to determine the concentrations of citrullinated chemokines in biologic fluid. Ninety-six-well plates (Thermo Fisher Scientific) were coated overnight at room temperature with mouse anti-human ENA-78/CXCL5, mouse anti-human MCP-1/CCL2, or goat anti-human MIP-1 $\alpha$ / CCL3 (R\&D Systems). Between each step, the plates were washed with wash buffer $(0.05 \%$ Tween 20 in phosphate buffered saline [PBS]). The plates were blocked with $1 \%$ bovine serum albumin (BSA) in PBS for 1 hour at room temperature and incubated with serum, SF, or standards for 2 hours at room temperature. Citrullinated recombinant human chemokines and native (noncitrullinated) recombinant human chemokines purified from the transfected HEK $293 \mathrm{~T}$ cells were used as standards and negative controls, respectively, for the ELISA. The samples were crosslinked onto the plates with $1 \%$ glutaraldehyde in PBS for 30 minutes at room temperature.

The plates were then incubated with $0.2 M$ Tris $\mathrm{HCl}$ (pH 7.8) for 30 minutes at room temperature to block the crosslinking. The plates were then incubated overnight at $37^{\circ} \mathrm{C}$ in a citrulline-modification solution consisting of 2 parts solution $\mathrm{A}\left(0.025 \%\right.$ [weight/volume] $\mathrm{FeCl}_{3}, 4.6 \mathrm{M} \mathrm{H}_{2} \mathrm{SO}_{4}$, and $3.0 \mathrm{M}$ $\left.\mathrm{H}_{3} \mathrm{PO}_{4}\right), 1$ part solution $\mathrm{B}(1 \%$ diacetylmonoxime, $0.5 \%$ antipyrine, and $1 M$ acetic acid), and 1 part $\mathrm{H}_{2} \mathrm{O}$ (18). The plates were incubated for 2 hours at room temperature with rabbit anti-modified citrulline (Millipore), diluted 1:2,500 in PBS containing $1 \%$ BSA. The plates were incubated for 2 hours at room temperature with horseradish peroxidase (HRP)-conjugated swine anti-rabbit $\operatorname{IgG}$ (Dako), diluted 1:1,000 in PBS containing 1\% BSA. Biotin-Tyramide Reagent (PerkinElmer), diluted $1: 1,000$ in $0.05 M$ Tris (base $\mathrm{pH} 8.5$ ), was added, followed by HRP-conjugated streptavidin. The plates were developed using tetramethylbenzidine, development was stopped with $2 \mathrm{~N} \mathrm{H}_{2} \mathrm{SO}_{4}$, and the plates were read on a microplate reader at $450 \mathrm{~nm}$. Serum and SF chemokine concentrations were also measured using DuoSet ELISA kits.
In vitro monocyte and polymorphonuclear neutrophil (PMN) chemotaxis assays. Monocytes and PMNs were isolated from normal human peripheral blood. Monocyte and PMN chemotaxis assays were performed using 48-well modified Boyden chambers (Neuro Probe) as described previously $(21,22)$. Noncitrullinated chemokines (OriGene), citrullinated chemokines, and reaction buffer consisting of $40 \mathrm{~m} M$ Tris $\mathrm{HCl}$, $2 \mathrm{mM} \mathrm{CaCl}, 250 \mathrm{n} M$ rabbit skeletal PAD, and $0.1 \%$ TFA were diluted by PBS with calcium and magnesium in the same manner and tested for chemotaxis. The composition of the reaction buffer that contained PAD enzymes was the same as the composition of the stock solution of citrullinated chemokines. PBS with calcium and magnesium and fMLP (100 $\mathrm{n} M)$ (Sigma-Aldrich) were used as negative and positive stimuli, respectively. For the inhibitor studies, monocytes $(2.5 \times$ $10^{6} / \mathrm{ml}$ ) were pretreated with $500 \mathrm{ng} / \mathrm{ml}$ pertussis toxin (SigmaAldrich), which is a $\mathrm{G}$ protein-coupled receptor antagonist, 10 $\mu \mathrm{g} / \mathrm{ml}$ anti-CXCR1, anti-CXCR2, or isotype control IgG2a (R\&D Systems) in PBS with calcium and magnesium for 1 hour to examine whether $G$ protein-coupled receptors are used by citrullinated ENA-78/CXCL5 to induce monocyte chemotaxis.

Mouse model of inflammatory arthritis. Female C57BL/6 mice (8-10 weeks old) were purchased from the National Cancer Institute. The mice were divided into the following 3 treatment groups: PBS, noncitrullinated ENA-78/ CXCL5 (Origene), and citrullinated ENA-78/CXCL5. The mice were anesthetized, and the knee circumference was determined by caliper measurements before intraarticular injection and calculated using the following formula: circumference $=\pi(a+b) / 2$, where $a$ is the laterolateral diameter and $b$ is the anteroposterior diameter. The anesthetized mice received $20 \mu \mathrm{l} / \mathrm{knee}$ joint of PBS, noncitrullinated ENA-78/ CXCL5 (16 ng), or citrullinated ENA-78/CXCL5 (16 ng). In all of the mice, circumference measurements were obtained in a blinded manner 24 hours after the intraarticular injection.

Hematoxylin and eosin (H\&E) and immunofluorescence staining. Mouse knee joints embedded in OCT compound were frozen and cut $(10 \mu \mathrm{m})$. H\&E staining was performed as described previously (23). Immunofluorescence staining was performed on cryosections from mouse knee joints to determine monocyte/macrophages, using rat antimouse $\mathrm{F} 4 / 80$ antibodies (GeneTex) at $1 \mu \mathrm{g} / \mathrm{ml}$ as primary antibody and Alexa Fluor 555-conjugated goat anti-rat IgG (Life Technologies) at a dilution of 1:200 as secondary antibody. The method of immunofluorescence staining has been described previously (21). The number of F4/80-positive monocyte/macrophages was calculated as the average of the number of cells in 3 fields $(400 \times)$ that showed the most remarkable infiltrates in the joint space where the injections were administered.

Statistical analysis. Statistical differences between experimental groups were determined by Student's $t$-test or one-way analysis of variance followed by Tukey's multiple comparison test for post hoc analysis. The clinical correlation between chemokine levels and clinical data was assessed by Pearson's correlation coefficient. Statistical analysis was performed with assistance from personnel at the Center for Statistical Consultation and Research at the University of Michigan. Results are expressed as the mean \pm SEM. $P$ values less than 0.05 were considered significant. 


\section{RESULTS}

\section{Detection of citrullinated rhENA-78/CXCL5 and} verification of in vitro citrullination of ENA-78/CXCL5. As shown in Figure 1A, the standard curve of citrullinated rhENA-78/CXCL5, as determined by ELISA, demonstrated high correlation between the concentration of citrullinated rhENA-78/CXCL5 and absorbance (Figure 1A). Noncitrullinated ENA-78/CXCL5 (1000 $\mathrm{pg} / \mathrm{ml}$ ) was not detected by this ELISA. We then performed Western blotting to confirm that rhENA-78/ CXCL5 was citrullinated in vitro. Citrullinated ENA-78/ CXCL5 was recognized by anti-modified citrulline antibody, while noncitrullinated ENA-78/CXCL5 was not (Figure 1B). The diagnostic +1Da mass shift occurring upon citrullination of ENA-78/CXCL5, which has 2 arginine residues, was identified by mass spectrometry (20). The mass spectrometry data indicated that both arginine residues were detected and citrullinated (Figures $1 \mathrm{C}$ and D). Citrullination of MCP-1/CCL2 and MIP- $1 \alpha /$ CCL3, which have 4 and 3 arginine residues, respectively, was also identified by mass spectrometry (data not shown).

Citrullinated chemokine concentrations in RA sera and normal control sera. To ascertain whether citrullinated chemokines were detectable in biologic fluids, we assayed serum samples obtained from patients with RA and normal control subjects, using a citrullinated chemokine sandwich ELISA. In normal control subjects and patients with RA, the mean \pm SEM levels of citrullinated ENA-78/CXCL5 were $1.2 \pm 0.7 \mathrm{pg} / \mathrm{ml}$ and $286 \pm 68 \mathrm{pg} / \mathrm{ml}$, respectively (Figure 2A). The levels of citrullinated MIP- $1 \alpha /$ CCL3 in normal and RA sera were $1.2 \pm 1.2 \mathrm{pg} / \mathrm{ml}$ and $624 \pm 96 \mathrm{pg} / \mathrm{ml}$, respectively (Figure 2B), and the levels of citrullinated MCP-1/CCL2 in normal and RA sera were $0.9 \pm 0.5 \mathrm{pg} / \mathrm{ml}$ and $143 \pm$ $18 \mathrm{pg} / \mathrm{ml}$, respectively (Figure 2C). The levels of citrullinated ENA-78/CXCL5, MIP-1 $\alpha /$ CCL3, and MCP-1/ CCL2 in patients with RA were all significantly higher than the levels in normal control subjects $(P<0.05)$. The mean \pm SEM concentrations of ENA-78/CXCL5
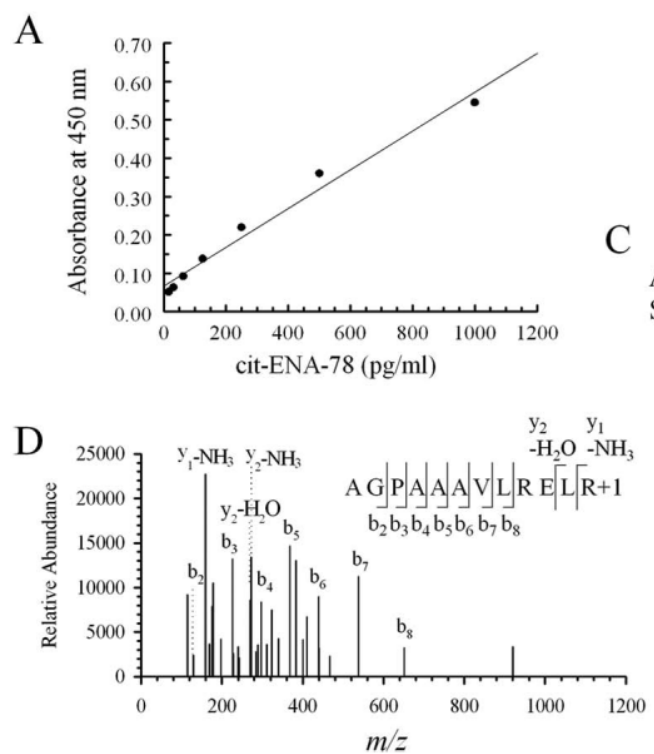

C
B

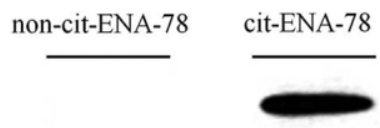
AGPAAAVLRELRCVCLQTTQGVHPKMISNLQVFAIGPQC SKVEVVASLKNGKEICLDPEAPFLKKVIQKILDGGNKEN

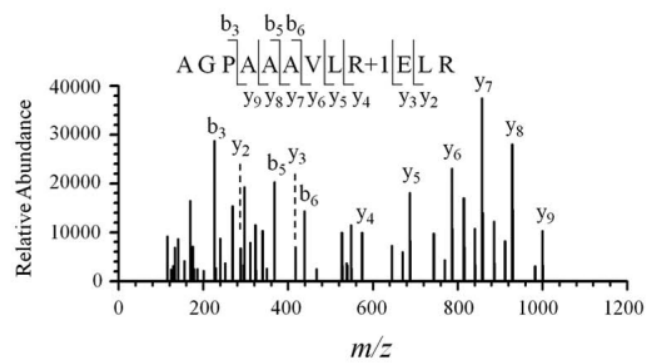

Figure 1. Detection of citrullinated recombinant human epithelial neutrophil-activating peptide 78 (ENA-78)/CXCL5 and verification of successful in vitro citrullination of ENA-78/CXCL5. A, Standard curve of citrullinated recombinant human ENA-78/CXCL5 as determined by enzyme-linked immunosorbent assay. Each point was determined using duplicate assays. B, Immunoblots showing that citrullinated recombinant human ENA-78/CXCL5 was recognized by anti-modified citrulline antibody (right) while noncitrullinated ENA-78/CXCL5 was not (left). The blot used to detect citrullinated protein was chemically modified prior to immunostaining. C, Amino acid sequence of ENA-78/CXCL5 (mature form 37-114). The boxed areas show the citrullinated arginines that were detected. D, Annotated tandem mass spectrometry (MS/MS) fragmentation spectra for the citrullinated ENA-78/CXCL5 peptides, showing the citrullinated arginine residues. Left, MS/MS spectrum confirming the presence of C-terminal citrullination at position 12 (R48) from the $\mathrm{y}_{1}$ and $\mathrm{y}_{2}$ ions present in the spectrum. Right, The presence of ions $\mathrm{y}_{4}$ to $\mathrm{y}_{9}$ in the spectrum confirms the presence of the "middle" $\mathrm{R}+1$ (citrullination) at position 9 (R45). The MS/MS fragmentation data were annotated using Expert System (Max-Planck Institute of Biochemistry). The precursor ion was observed with a mass error of $<1$ parts per million, and the error for the fragment ions was $\leq 0.02$ daltons. 
A

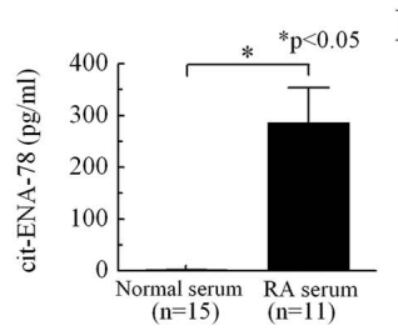

D

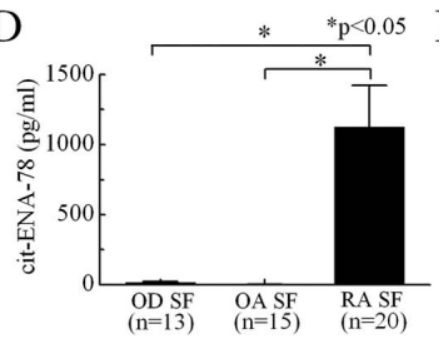

B

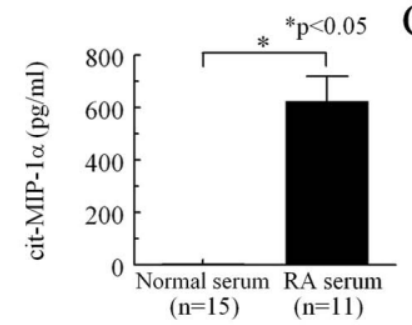

E

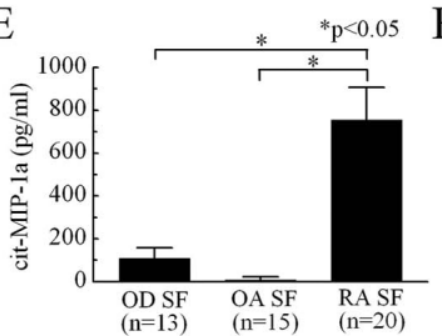

C

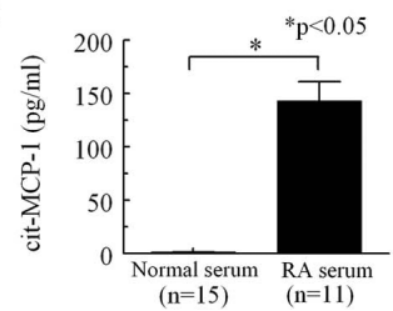

F

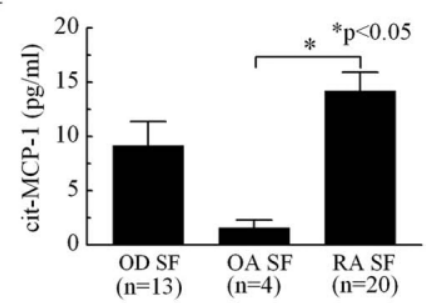

Figure 2. Expression of citrullinated chemokines in rheumatoid arthritis (RA) sera and synovial fluid (SF). Citrullinated chemokines were measured using an enzyme-linked immunosorbent assay (ELISA) in which chemokines were captured on an ELISA plate, followed by detection with anti-modified citrulline after chemical modification of citrulline residues. Rheumatoid factor was depleted from RA SF and RA sera prior to the ELISA. A-C, Concentrations of citrullinated epithelial neutrophil-activating peptide 78 (ENA-78)/CXCL5 (A), macrophage inflammatory protein $1 \alpha(\mathrm{MIP}-1 \alpha) / \mathrm{CCL} 3(\mathbf{B})$, and monocyte chemotactic protein 1 (MCP-1)/CCL2 (C) in normal sera and sera from patients with RA. D-F, Concentrations of citrullinated ENA-78/CXCL5, citrullinated MIP-1 $\alpha / C C L 3$, and citrullinated MCP-1/CCL2 in SF from patients with other inflammatory rheumatic diseases (OD), patients with osteoarthritis (OA), and patients with RA. Bars show the mean \pm SEM.

were $3,165 \pm 825 \mathrm{pg} / \mathrm{ml}$ in normal sera and 3,942 $\pm 1,139$ $\mathrm{pg} / \mathrm{ml}$ in RA sera, the levels of MIP- $1 \alpha / \mathrm{CCL} 3$ were $230 \pm 176 \mathrm{pg} / \mathrm{ml}$ and $727 \pm 420 \mathrm{pg} / \mathrm{ml}$, respectively, and the levels of MCP-1/CCL2 were $43 \pm 22 \mathrm{pg} / \mathrm{ml}$ and $289 \pm 271 \mathrm{pg} / \mathrm{ml}$, respectively; none of the differences between groups were statistically significant (results not shown).

Citrullinated chemokine concentrations in SF from patients with RA, patients with $\mathrm{OA}$, and patients with other rheumatic diseases. The concentrations of citrullinated chemokines in SF samples obtained from patients with RA, patients with OA, and patients with other inflammatory rheumatic diseases were measured by sandwich ELISA. The mean \pm SEM concentration of citrullinated ENA-78/CXCL5 was significantly higher in SF from patients with RA $(1,126 \pm 297 \mathrm{pg} / \mathrm{ml})$ than in SF from patients with $\mathrm{OA}(2.3 \pm 1.0 \mathrm{pg} / \mathrm{ml})$ and patients with other inflammatory rheumatic diseases $(15 \pm 9$ $\mathrm{pg} / \mathrm{ml})(P<0.05)$ (Figure 2D). The mean \pm SEM concentration of citrullinated MIP-1 $\alpha /$ CCL3 was also significantly higher in the SF of patients with RA (755 \pm $152 \mathrm{pg} / \mathrm{ml}$ ) compared with that in the SF of patients with other inflammatory rheumatic diseases $(109 \pm 49 \mathrm{pg} / \mathrm{ml})$ and patients with OA $(9 \pm 15 \mathrm{pg} / \mathrm{ml})(P<0.05)$ (Figure 2E). Citrullinated MCP-1/CCL2 concentrations were significantly higher in RA SF $(14 \pm 2 \mathrm{pg} / \mathrm{ml})$ compared with OA SF $(1.6 \pm 0.7 \mathrm{pg} / \mathrm{ml})(P<0.05)$ (Figure $2 \mathrm{~F})$.

We also used ELISA kits to measure chemokine concentrations. The mean \pm SEM concentrations of ENA-78/CXCL5 were $163 \pm 107 \mathrm{pg} / \mathrm{ml}$ in RA SF, $0 \pm 0$ $\mathrm{pg} / \mathrm{ml}$ in the SF of patients with other diseases, and $19 \pm$ $13 \mathrm{pg} / \mathrm{ml}$ in OA SF. The concentrations of MIP- $1 \alpha /$ CCL3 were $460 \pm 171 \mathrm{pg} / \mathrm{ml}$ in RA SF, $31 \pm 10 \mathrm{pg} / \mathrm{ml}$ in $\mathrm{SF}$ from patients with other rheumatic diseases, and $75 \pm 52 \mathrm{pg} / \mathrm{ml}$ in OA SF. The concentrations of MCP$1 / \mathrm{CCL} 2$ were $1,085 \pm 398 \mathrm{pg} / \mathrm{ml}$ in RA SF, $288 \pm 42$ $\mathrm{pg} / \mathrm{ml}$ in SF from patients with other rheumatic diseases, and $390 \pm 80 \mathrm{pg} / \mathrm{ml}$ in OA SF. There were no significant differences in SF chemokine concentrations between these groups.

Positive correlation between citrullinated ENA78/CXCL5 concentrations and the C-reactive protein (CRP) level and erythrocyte sedimentation rate (ESR) in RA SF. We analyzed the relationship between the citrullinated chemokine concentrations in SF and clinical data in patients with RA. As shown in Table 1, the clinical characteristics of these patients were as follows: mean \pm SEM age $52.7 \pm 3.4$ years, disease duration $145.7 \pm 39.2$ months, ESR $38.1 \pm 4.3 \mathrm{~mm} /$ hour, CRP level $40.5 \pm 9.9 \mathrm{mg} /$ liter, Disease Activity Score in 28 
Table 1. Clinical characteristics of the patients with rheumatoid arthritis whose synovial fluid was studied*

\begin{tabular}{lc}
\hline Male/female, $\%(\mathrm{n}=20)$ & $20 / 80$ \\
Age, years $(\mathrm{n}=20)$ & $52.7 \pm 3.4$ \\
Disease duration, months $(\mathrm{n}=20)$ & $145.7 \pm 39.2$ \\
Tender joint count in 28 joints $(\mathrm{n}=13)$ & $5.7 \pm 2.1$ \\
Swollen joint count in 28 joints $(\mathrm{n}=13)$ & $4.5 \pm 1.9$ \\
Bone erosion, yes/no, \% $(\mathrm{n}=20)$ & $40 / 60$ \\
ESR, mm/hour $(\mathrm{n}=15)$ & $38.1 \pm 4.3$ \\
CRP, mg/liter $(\mathrm{n}=14)$ & $40.5 \pm 9.9$ \\
DAS28 $(\mathrm{n}=12)$ & $5.1 \pm 0.4$ \\
Rheumatoid factor, units/ml $(\mathrm{n}=10)$ & $74.8 \pm 43.2$ \\
Anti-CCP, AU/ml $(\mathrm{n}=10)$ & $2,296.1 \pm 1.559 .1$
\end{tabular}

* Except where indicated otherwise, values are the mean \pm SEM. ESR $=$ erythrocyte sedimentation rate; $\mathrm{CRP}=\mathrm{C}$-reactive protein; DAS28 $=$ Disease Activity Score in 28 joints; anti-CCP $=$ anti-cyclic citrullinated peptide.

joints (24) $5.1 \pm 0.4$, tender joint count $5.7 \pm 2.1$, swollen joint count $4.5 \pm 1.9$, RF level $74.8 \pm 43.2$ units/ml, and anti-CCP antibody level 2,296.1 \pm 1,559.1 AU/ml. As shown in Figure 3, citrullinated ENA-78/ CXCL5 concentrations were significantly correlated with CRP levels $(\mathrm{r}=0.69, P<0.05)$ and the ESR $(\mathrm{r}=$ $0.77, P<0.05)$, and citrullinated MCP-1/CCL2 concentrations were significantly correlated with the ESR $(\mathrm{r}=$ $0.64, P<0.05)$. There was no significant correlation between the concentration of citrullinated MIP-1 $\alpha /$ CCL3 in RA SF with the other clinical parameters examined.

Citrullinated ENA-78/CXCL5-induced monocyte migratory activity via CXCR1 and CXCR2. Under normal conditions, ENA-78/CXCL5 induces PMN migration, but it does not induce monocyte migration $(10,25,26)$. We performed monocyte and PMN chemotaxis assays using a modified Boyden chamber, and the results showed that the fold increase in monocyte migration was significantly higher in response to citrullinated ENA-78/ CXCL5 at $0.1 \mathrm{n} M, 1 \mathrm{n} M$, and $10 \mathrm{n} M$ (mean \pm SEM $1.6 \pm$ $0.1,1.9 \pm 0.2$, and $1.9 \pm 0.2$, respectively) than in response to noncitrullinated ENA-78/CXCL5 and PBS (Figure 4A). We also tested various concentrations of reaction buffer in citrullinated ENA-78/CXCL5 stock solution for chemotaxis of monocytes. The reaction buffer including PAD enzymes did not induce monocyte migration at the concentrations tested. In contrast to citrullinated ENA-78/CXCL5, citrullinated MCP-1/ CCL2 recruited significantly fewer monocytes at $10 \mathrm{n} M$ and $100 \mathrm{n} M$ than did noncitrullinated MCP-1/CCL2 (data not shown), and citrullinated MIP- $1 \alpha /$ CCL3 tended to have the same level of monocyte-recruiting activity as noncitrullinated MIP- $1 \alpha /$ CCL3 at $0.01-100$ $\mathrm{n} M$ (data not shown).

PMN chemotaxis assays showed that noncitrulli- nated ENA-78/CXCL5 had significantly higher PMN chemotactic activity at $0.1 \mathrm{n} M(1.5 \pm 0.2)$ and $1 \mathrm{n} M$ $(1.6 \pm 0.2)$ compared with PBS $(P<0.05)$, while citrullinated ENA-78/CXCL5 had significantly higher activity at $10 \mathrm{n} M(1.4 \pm 0.1)$ compared with PBS $(P<$ 0.05 ) (Figure 4B). Thus, the fold increase in PMN

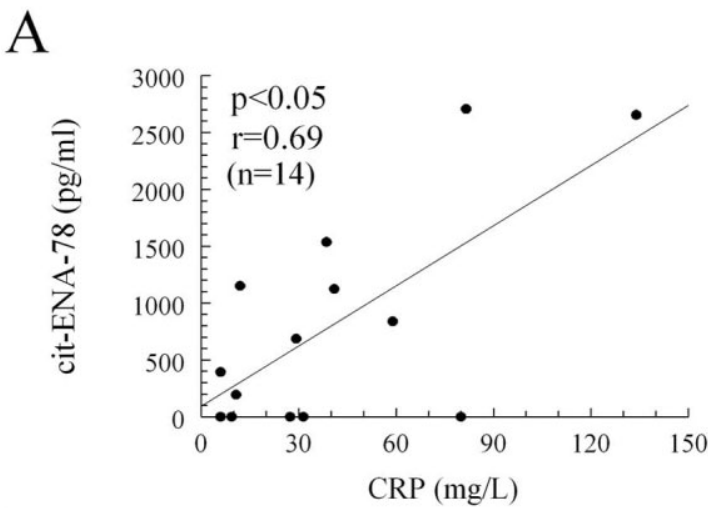

B

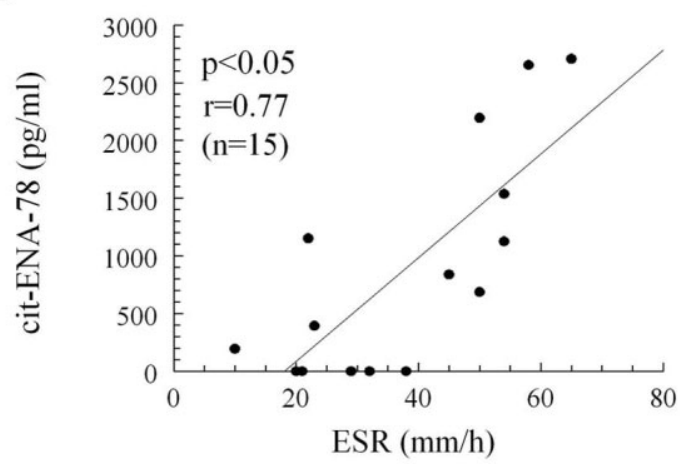

C

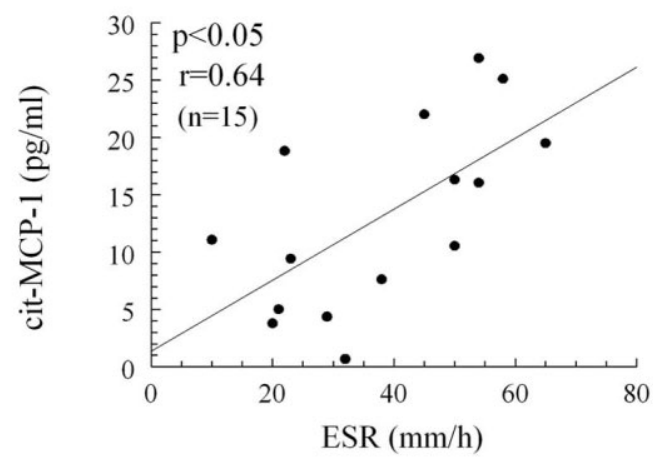

Figure 3. Correlation of clinical data with citrullinated chemokine levels in RA SF. Positive correlations between citrullinated ENA-78/ CXCL5 and the C-reactive protein (CRP) level (A), citrullinated ENA-78/CXCL5 and the erythrocyte sedimentation rate (ESR) (B), and citrullinated MCP-1/CCL2 and the ESR (C) were observed. See Figure 2 for other definitions. 
A

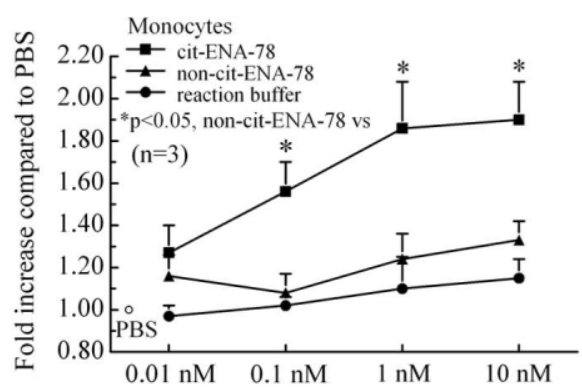

$\mathrm{C}$

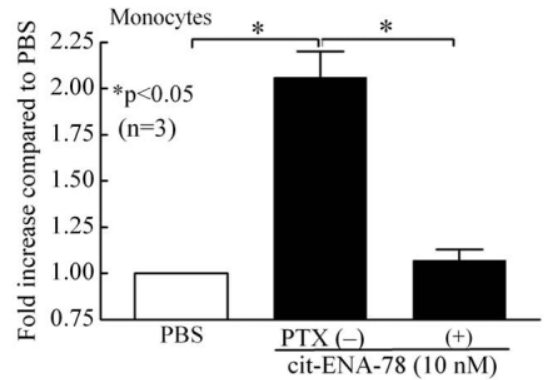

B

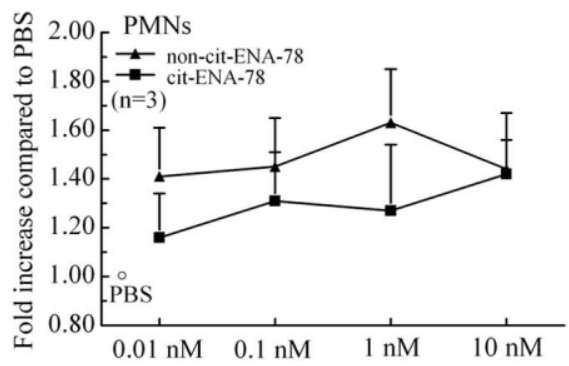

$\mathrm{D}$

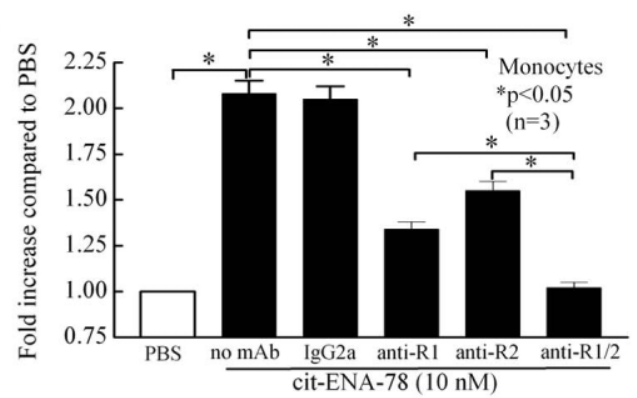

Figure 4. Monocyte recruitment by citrullinated epithelial neutrophil-activating peptide 78 (ENA-78)/CXCL5. A, Fold increase in monocyte migration in response to citrullinated ENA-78/CXCL5 and noncitrullinated ENA-78/CXCL5, as determined by monocyte chemotaxis assay. At 0.1 $\mathrm{n} M, 1 \mathrm{n} M$, and $10 \mathrm{n} M$, the response to citrullinated ENA-78/CXCL5 was significantly higher than the response to noncitrullinated ENA-78/CXCL5 and phosphate buffered saline (PBS). As controls, various concentrations of reaction buffer including peptidylarginine deiminase enzymes were tested for chemotaxis of monocytes. There was no significant difference between the response to reaction buffer and the response to PBS. The mean \pm SEM fold increase in monocyte migration in response to fMLP $(100 \mathrm{n} M)$ was $4.4 \pm 0.5$. B, Fold increase in polymorphonuclear neutrophil (PMN) migration in response to citrullinated ENA-78/CXCL5 and noncitrullinated ENA-78/CXCL5, as determined by chemotaxis assay. At either $0.1 \mathrm{n} M$ or $1 \mathrm{n} M$, the response to noncitrullinated ENA-78/CXCL5 was significantly higher than the response to PBS $(P<0.05)$. The response to citrullinated ENA-78/CXCL5 was lower than the response to noncitrullinated ENA-78/CXCL5, but the difference was not significant. The mean \pm SEM fold increase in PMN migration in response to fMLP $(100 \mathrm{n} M)$ was $7.5 \pm 2.5$. C, Effect of pertussis toxin (PTX) on the increased monocyte migration in response to citrullinated ENA-78/CXCL5. D, Effect of anti-CXCR1 (anti-R1) and anti-CXCR2 (anti-R2) on the increased monocyte migration in response to citrullinated ENA-78/CXCL5. All assays were performed in quadruplicate, with 3 high-power fields (400×) counted in each replicate well. The fold increase was determined by dividing the number of cells migrated by the number of cells migrated to the negative control PBS. Values are the mean \pm SEM.

migration in response to citrullinated ENA-78/CXCL5 tended to be lower than that in response to noncitrullinated ENA-78/CXCL5. However, there was no significant difference in PMN chemotaxis between noncitrullinated and citrullinated ENA-78/CXCL5.

In monocyte chemotaxis assays using pertussis toxin (a $\mathrm{G}$ protein-coupled receptor antagonist), antiCXCR1, and anti-CXCR2 antibodies, the fold increase in the response to citrullinated ENA-78/CXCL5 with pertussis toxin was significantly lower (mean \pm SEM $1.07 \pm 0.06)$ compared with that without pertussis toxin $(2.06 \pm 0.14 ; P<0.05)$ (Figure $4 \mathrm{C})$. We then examined whether monocyte migration is mediated via the $\mathrm{G}$ protein-coupled receptors CXCR1 and CXCR2. The fold increases in the response to citrullinated ENA-78/ CXCL5 with anti-CXCR1 or anti-CXCR2 antibodies were significantly lower $(1.34 \pm 0.04$ and $1.55 \pm 0.05$, respectively) compared with that without the antibodies $(2.05 \pm 0.07)$, and the fold increase in the response to citrullinated ENA-78/CXCL5 with a combination of anti-CXCR1 and anti-CXCR2 antibodies was significantly lower $(1.02 \pm 0.03)$ compared with that without the antibodies, with anti-CXCR1, or with anti-CXCR2 $(P<0.05)$. IgG2a was used as an isotype-matched control monoclonal antibody that did not inhibit monocyte migration (Figure 4D). These results indicated that citrullinated ENA-78/CXCL5 induced monocyte migration via CXCR1 and CXCR2.

Citrullinated ENA-78/CXCL5-enhanced induction of inflammatory arthritis and recruitment of monocyte/macrophages in vivo. To test the inflammatory activity of citrullinated ENA-78/CXCL5 in vivo, we 
A
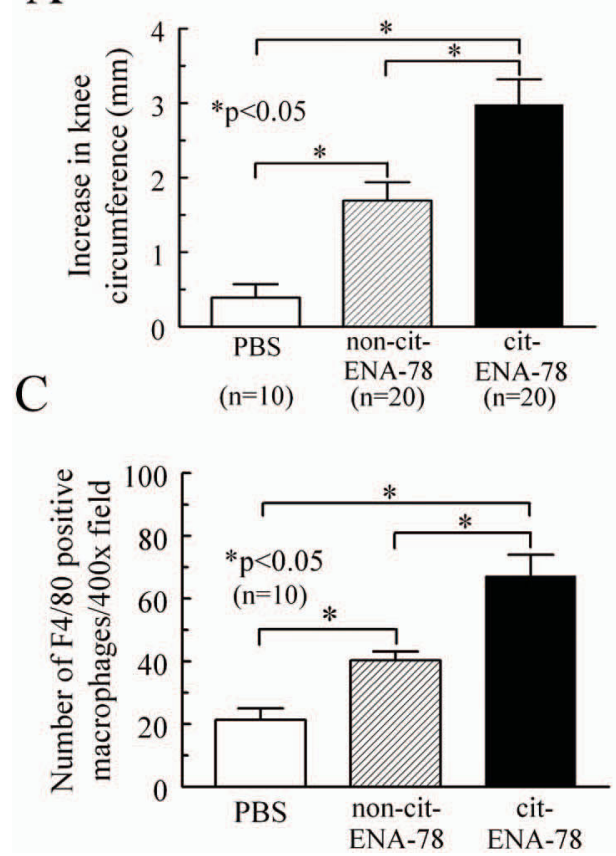

B

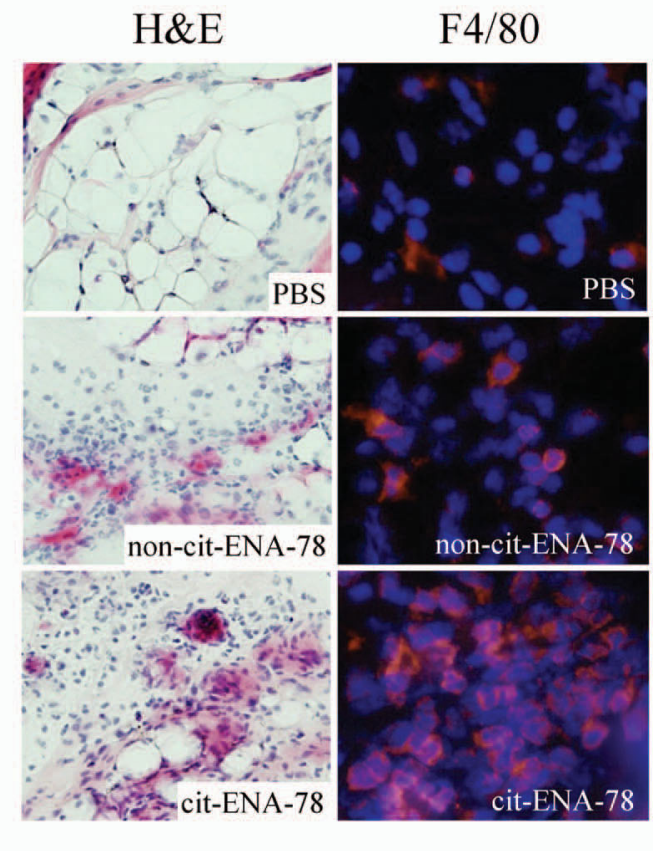

Figure 5. Effect of citrullinated ENA-78/CXCL5 in vivo. The knees of female C57BL/6 mice were injected intraarticularly with PBS, noncitrullinated ENA-78/CXCL5, or citrullinated ENA-78/CXCL5 to induce inflammation. A, Circumference of the mouse knees 24 hours after administration of the intraarticular injections. The increases in knee circumference were significantly greater in mice that received citrullinated ENA-78/CXCL5 compared with those that received PBS or noncitrullinated ENA-78/CXCL5 and were also significantly greater in mice that received noncitrullinated ENA-78/CXCL5 compared with those that received PBS. B, Left, Hematoxylin and eosin (H\&E)-stained mouse knee joint sections, showing inflammatory cell infiltration in all groups and severe inflammation in mice treated with citrullinated ENA-78/CXCL5. Right, Immunofluorescence-stained mouse knee joint sections, showing a greater number of F4/80-positive monocyte/macrophages in mice treated with citrullinated ENA-78/CXCL5. Fluorescent red and blue staining show F4/80-positive monocyte/macrophages and cell nuclei, respectively. Original magnification $\times 400$. C, Numbers of F4/80-positive monocyte/macrophages in mice treated with PBS, noncitrullinated ENA-78/CXCL5, and citrullinated ENA-78/CXCL5. Values are the mean \pm SEM ( $n$ values are the number of joints). See Figure 4 for other definitions.

injected PBS, noncitrullinated ENA-78/CXCL5, or citrullinated ENA-78/CXCL5 into mouse knee joints. Joints injected with citrullinated ENA-78/CXCL5 had a significantly greater increase in circumference (mean \pm SEM $3.0 \pm 0.3 \mathrm{~mm}$ ), and hence joint swelling, compared with knees injected with PBS $(0.4 \pm 0.2 \mathrm{~mm} ; P<0.05)$ or noncitrullinated ENA-78/CXCL5 $(1.7 \pm 0.3 \mathrm{~mm} ; P<$ 0.05). Joints injected with noncitrullinated ENA-78/ CXCL5 had significantly increased joint swelling compared with joints injected with PBS $(P<0.05)$ (Figure $5 \mathrm{~A})$. H\&E staining showed inflammatory cell infiltration in each treatment group, and inflammation was especially severe in the group that received citrullinated ENA-78/CXCL5 (Figure 5B). Immunofluorescence staining revealed that the number of F4/80-positive monocyte/macrophages was significantly higher in mouse knee joints injected with citrullinated ENA-78/ CXCL5 (mean \pm SEM $67 \pm 7$ macrophages) compared with joints injected with noncitrullinated ENA-78/
CXCL5 (40 \pm 3 macrophages) or PBS $(21 \pm 4$ macrophages). In addition, the number of F4/80-positive monocyte/macrophages was significantly higher in the mice treated with noncitrullinated ENA-78/CXCL5 compared with those that received PBS $(P<0.05)$ (Figures 5B and C).

\section{DISCUSSION}

We observed the presence of citrullinated ENA$78 /$ CXCL5, MIP- $1 \alpha /$ CCL3, and MCP-1/CCL2 in RA sera and RA SF. There is evidence that some chemokines can be citrullinated in vitro (27-30); however, not all cytokines can be citrullinated. Proost et al showed that PAD efficiently and site-specifically citrullinated ENA-78/CXCL5 and IL-8/CXCL8 but not IL-1 $\beta$ (27). Struyf et al demonstrated that stromal cell-derived factor 1 (SDF-1)/CXCL12 can be citrullinated in vitro, and that SDF-1/CXCL12 and PAD were coexpressed in 
the colon tissue of patients with Crohn's disease (30). However, those investigators did not directly demonstrate the presence of citrullinated SDF-1/CXCL12 in diseased colon tissue.

In the current study, we demonstrated that the concentrations of citrullinated ENA-78/CXCL5, MIP$1 \alpha /$ CCL3, and MCP-1/CCL2 were all significantly higher in RA sera than in normal control sera. Furthermore, citrullinated ENA-78/CXCL5 and MIP- $1 \alpha /$ CCL3 concentrations were significantly higher in RA SF than in $\mathrm{SF}$ from patients with $\mathrm{OA}$ and patients with other rheumatic diseases, and the level of citrullinated MCP$1 / C C L 2$ was significantly higher in RA SF than in OA SF. In particular, the concentration of citrullinated ENA-78/CXCL5 was high in RA SF but was barely detected in SF from patients with OA or other rheumatic diseases. Additionally, the PAD4 level measured by ELISA is significantly elevated in RA SF compared with SF from patients with OA and patients with ankylosing spondylitis (31). This fact supports the notion that chemokines, including ENA-78/CXCL5, are citrullinated more efficiently in RA joints than in the inflamed joints of patients with other diseases. In our study, the concentrations of ENA-78/CXCL5 and MIP- $1 \alpha /$ CCL3 in $\mathrm{SF}$ as measured by standard ELISA kits ( $R \& D$ Systems) were lower than the concentrations of citrullinated ENA-78/CXCL5 and MIP- $1 \alpha /$ CCL3 in SF as measured by the new ELISA system developed by our group. We observed that after citrullination, purified chemokines were not detected by standard ELISAs (data not shown). It is possible that these ELISAs may not completely recognize citrullinated chemokines in RA SF.

We showed that among 3 citrullinated chemokines tested in RA SF, the concentrations of citrullinated ENA-78/CXCL5 correlated most closely with clinical data (the CRP level and the ESR). Chemokines are readily detectable in RA SF $(11,32-36)$, and the concentrations of MIP- $1 \alpha /$ CCL3 and IL-8/CXCL8 in SF correlate with CRP levels in serum $(35,36)$. In our study, we observed significant positive correlations between citrullinated ENA-78/CXCL5 and not only the CRP level but also the ESR, whereas the ENA-78/CXCL5 concentration did not correlate with any clinical parameters examined (data not shown). These results suggest that compared with the other chemokines that we examined, citrullinated ENA-78/CXCL5 is more closely related to disease activity in RA.

Citrullination has been reported to decrease the activities of certain chemokines in some instances (37). In vitro, ENA-78/CXCL5 (0.1-10 $\mathrm{n} M)$ induces PMN migration in a dose-responsive manner $(10,25)$. However, citrullination of ENA-78/CXCL5 has been reported to reduce in vitro and in vivo PMN chemotaxis, intracellular calcium signaling, phosphorylation of extracellular signal-regulated kinase, and internalization of CXCR2 compared with noncitrullinated ENA-78/ CXCL5 (38). We showed that citrullination of ENA-78/ CXCL5 directly supports monocyte migration but not PMN migration. These results indicate the possibility that citrullination of ENA-78/CXCL5 does not increase the function of ENA-78/CXCL5 as a PMN recruiter but instead dampens it.

Surprisingly, we observed that citrullinated ENA78/CXCL5 acquired a monocyte-recruiting function that noncitrullinated ENA-78/CXCL5 did not have. Normally, ENA-78/CXCL5 is not a potent chemotactic factor for monocytes but rather for PMNs and endothelial cells and also possesses angiogenic properties $(10,25,39,40)$. Monocyte chemotaxis assays showed that citrullinated ENA-78/CXCL5 recruited monocytes in a dose-dependent manner, while noncitrullinated ENA78/CXCL5 did not. These results suggest that citrullination of ENA-78/CXCL5 results in its conversion from a non-monocyte-recruiting chemokine to a monocyterecruiting chemokine.

We then investigated whether citrullinated ENA78/CXCL5 recruited monocytes through the G proteincoupled receptors CXCR2, which is the primary ENA78/CXCL5 receptor, and CXCR1, which has 78\% homology with CXCR2 at the amino acid level $(41,42)$. Citrullinated ENA-78-induced monocyte migration was completely inhibited by pertussis toxin (a G proteincoupled receptor antagonist) or by a combination of anti-CXCR1 and anti-CXCR2 antibodies. These results suggest that citrullinated ENA-78/CXCL5 recruits monocytes via both CXCR1 and CXCR2. ENA-78/ CXCL5 elicits PMN chemotaxis by interacting with the chemokine receptor CXCR2 present on the PMN cell surface $(43,44)$. This receptor is expressed on many different cells including PMNs and monocytes (45). CXCR1 is also present on the surface of human monocytes $(26,46)$. CXCR1 and CXCR2 are expressed on $23-90 \%$ and $22-93 \%$ of human monocytes, respectively (26). IL-8/CXCL8, neutrophil-activating peptide/ CXCL7, growth-related oncogene $\alpha /$ CXCL1, and ENA78/CXCL5 bind to CXCR2 with high affinity, whereas IL-8/CXCL8 exhibits high affinity for CXCR1 as well $(47,48)$. Our results suggest that citrullination may increase the affinity of ENA-78/CXCL5 for CXCR1 (38).

We clearly show that at physiologically relevant concentrations, citrullinated ENA-78/CXCL5 recruits 
monocytes. We also show data confirming that citrullinated ENA-78/CXCL5 preferentially binds to the CXCR1 receptor instead of the normal receptor for ENA-78/CXCL5, namely CXCR2. This indicates that modification of ENA-78/CXCL5 changes its receptorbinding affinity (and likely its conformation). This also explains, at least in part, the ability of citrullinated ENA-78/CXCL5 to recruit monocytes. Nonetheless, assuming that both citrullinated ENA-78/CXCL5 and noncitrullinated ENA-78/CXCL5 are present in SF and are binding to the same receptor, the modified or unmodified forms of ENA-78/CXCL5 that are present in greater quantities would be the forms that are more competitive for binding the available receptor. However, we now present evidence that citrullinated ENA-78/ CXCL5 utilizes an alternative receptor (CXCR1), indicating that the concentration of citrullinated ENA-78/ CXCL5 is likely more important when considering the monocyte-recruiting activity of citrullinated ENA-78/ CXCL5. We believe that the conversion of ENA-78/ CXCL5, a potent neutrophil recruitment factor to citrullinated ENA-78/CXCL5, an active monocyte recruitment factor, represents a change to a more chronic inflammatory response in RA-affected joints.

Last, we examined whether citrullination of ENA-78/CXCL5 results in increased inflammation and monocyte migration in vivo compared with noncitrullinated ENA-78/CXCL5. To test this hypothesis, we injected noncitrullinated or citrullinated ENA-78/CXCL5 into mouse knee joints and evaluated joint inflammation and monocyte migration. Citrullinated ENA-78/CXCL5 increased the knee circumference more than noncitrullinated ENA-78/CXCL5. Immunofluorescence staining showed that citrullinated ENA-78/CXCL5 induced more F4/80-positive monocyte/macrophage ingress into the synovial tissue of mouse knees compared with noncitrullinated ENA-78/CXCL5. These results suggest that citrullination of ENA-78/CXCL5 induces more severe inflammation in mouse knee joints through monocyte recruitment.

In the current study, although RA sera and RA SF were obtained from different groups of patients, the concentration of citrullinated ENA-78/CXCL5 in RA SF was higher than that in RA sera. These results suggest that citrullinated ENA-78/CXCL5 may be produced mainly in the joints and functions to recruit monocytes to the joints. Other citrullinated proteins (e.g, citrullinated vimentin) stimulate proinflammatoy cytokine production from fibroblast-like synoviocytes or peripheral blood mononuclear cells in RA $(49,50)$. It is possible that citrullinated ENA-78/CXCL5 may also stimulate cells other than monocytes in mouse knee joints to release inflammatory mediators and aggravate the inflammatory response.

In addition, our study demonstrated that noncitrullinated ENA-78/CXCL5 recruited more monocyte/ macrophages in vivo compared with PBS. In general, ENA-78/CXCL5 recruits neutrophils in vitro and in vivo. However, it is possible that other types of cells besides neutrophils respond to ENA-78/CXCL5 in vivo. It has been reported that macrophages, which are either inflammatory monocyte-derived cells or reemerging tissue-resident macrophages, increase in number during acute inflammatory responses (51). It is quite possible that the number of monocyte/macrophages increases in vivo during ENA-78/CXCL5-induced acute inflammation.

In conclusion, citrullinated ENA-78/CXCL5 was detected in RA SF and sera. The concentrations were significantly higher in SF from patients with RA than in SF from patients with OA or other inflammatory rheumatic diseases and correlated with the CRP level and the ESR. Citrullinated ENA-78/CXCL5 induced monocyte migration via CXCR1 and CXCR2 in vitro, while noncitrullinated ENA-78/CXCL5 did not. Mouse knees injected with citrullinated ENA-78/CXCL5 had more inflammation and monocyte/macrophages than did those injected with noncitrullinated ENA-78/CXCL5, suggesting that citrullination enhances the proinflammatory activity of ENA-78/CXCL5 and accelerates disease progression in inflammatory arthritis.

\section{ACKNOWLEDGMENTS}

We thank Dr. David A. Fox (Division of Rheumatology, University of Michigan Medical School) for helpful comments and interpretation of the results; Dr. Henriette A. Remmer (Proteomics \& Peptide Synthesis Core, University of Michigan Medical School) and Dr. Michael Ford (MS Bioworks) for helpful comments on the mass spectrometry data and for providing the spectra; and Ray A. Ohara (University of Michigan) for assistance performing the experiments.

\section{AUTHOR CONTRIBUTIONS}

All authors were involved in drafting the article or revising it critically for important intellectual content, and all authors approved the final version to be published. Dr. Yoshida had full access to all of the data in the study and takes responsibility for the integrity of the data and the accuracy of the data analysis.

Study conception and design. Yoshida, Korchynskyi, Tak, Ruth, Campbell, Amin, Koch.

Acquisition of data. Yoshida, Korchynskyi, Isozaki, Baeten, Gerlag, Amin.

Analysis and interpretation of data. Yoshida, Korchynskyi, Ruth, Campbell, Koch. 


\section{REFERENCES}

1. Tak PP, Bresnihan B. The pathogenesis and prevention of joint damage in rheumatoid arthritis: advances from synovial biopsy and tissue analysis. Arthritis Rheum 2000;43:2619-33.

2. Van Venrooij WJ, van Beers JJ, Pruijn GJ. Anti-CCP antibodies: the past, the present and the future. Nat Rev Rheumatol 2011;7: 391-8.

3. Schellekens GA, de Jong BA, van den Hoogen FH, van de Putte LB, van Venrooij WJ. Citrulline is an essential constituent of antigenic determinants recognized by rheumatoid arthritis-specific autoantibodies. J Clin Invest 1998;101:273-81.

4. Sebbag M, Moinard N, Auger I, Clavel C, Arnaud J, Nogueira L, et al. Epitopes of human fibrin recognized by the rheumatoid arthritis-specific autoantibodies to citrullinated proteins. Eur J Immunol 2006;36:2250-63.

5. Suzuki A, Yamada R, Yamamoto K. Citrullination by peptidylarginine deiminase in rheumatoid arthritis. Ann N Y Acad Sci 2007;1108:323-39.

6. Mangat P, Wegner N, Venables PJ, Potempa J. Bacterial and human peptidylarginine deiminases: targets for inhibiting the autoimmune response in rheumatoid arthritis? Arthritis Res Ther 2010;12:209.

7. Szekanecz Z, Kim J, Koch AE. Chemokines and chemokine receptors in rheumatoid arthritis. Semin Immunol 2003;15:15-21.

8. Haringman JJ, Ludikhuize J, Tak PP. Chemokines in joint disease: the key to inflammation? Ann Rheum Dis 2004;63:1186-94.

9. Zlotnik A, Yoshie O. Chemokines: a new classification system and their role in immunity. Immunity 2000;12:121-7.

10. Walz A, Burgener R, Car B, Baggiolini M, Kunkel SL, Strieter RM. Structure and neutrophil-activating properties of a novel inflammatory peptide (ENA-78) with homology to interleukin 8. J Exp Med 1991;174:1355-62.

11. Koch AE, Kunkel SL, Harlow LA, Mazarakis DD, Haines GK, Burdick MD, et al. Epithelial neutrophil activating peptide-78: a novel chemotactic cytokine for neutrophils in arthritis. J Clin Invest 1994;94:1012-8.

12. Halloran MM, Woods JM, Strieter RM, Szekanecz Z, Volin MV, Hosaka S, et al. The role of an epithelial neutrophil-activating peptide-78-like protein in rat adjuvant-induced arthritis. J Immunol 1999;162:7492-500.

13. Kinloch A, Tatzer V, Wait R, Peston D, Lundberg K, Donatien P, et al. Identification of citrullinated $\alpha$-enolase as a candidate autoantigen in rheumatoid arthritis. Arthritis Res Ther 2005;7: R1421-9.

14. Van Steendam K, Tilleman K, De Ceuleneer M, De Keyser F, Elewaut D, Deforce D. Citrullinated vimentin as an important antigen in immune complexes from synovial fluid of rheumatoid arthritis patients with antibodies against citrullinated proteins. Arthritis Res Ther 2010;12:R132.

15. Vossenaar ER, Smeets TJ, Kraan MC, Raats JM, van Venrooij WJ, Tak PP. The presence of citrullinated proteins is not specific for rheumatoid synovial tissue. Arthritis Rheum 2004;50:3485-94.

16. Foulquier C, Sebbag M, Clavel C, Chapuy-Regaud S, Al Badine R, Mechin MC, et al. Peptidyl arginine deiminase type 2 (PAD-2) and PAD-4 but not PAD-1, PAD-3, and PAD-6 are expressed in rheumatoid arthritis synovium in close association with tissue inflammation. Arthritis Rheum 2007;56:3541-53.

17. Korchynskyi O, ten Dijke P. Identification and functional characterization of distinct critically important bone morphogenetic protein-specific response elements in the Id1 promoter. J Biol Chem 2002;277:4883-91.

18. Senshu T, Sato T, Inoue T, Akiyama K, Asaga H. Detection of citrulline residues in deiminated proteins on polyvinylidene difluoride membrane. Anal Biochem 1992;203:94-100.

19. Tsuji Y, Akiyama M, Arita K, Senshu T, Shimizu H. Changing pattern of deiminated proteins in developing human epidermis J Invest Dermatol 2003;120:817-22.

20. Kubota K, Yoneyama-Takazawa T, Ichikawa K. Determination of sites citrullinated by peptidylarginine deiminase using $18 \mathrm{O}$ stable isotope labeling and mass spectrometry. Rapid Commun Mass Spectrom 2005;19:683-8.

21. Ruth JH, Haas CS, Park CC, Amin MA, Martinez RJ, Haines GK III, et al. CXCL16-mediated cell recruitment to rheumatoid arthritis synovial tissue and murine lymph nodes is dependent upon the MAPK pathway. Arthritis Rheum 2006;54:765-78.

22. Ruth JH, Arendt MD, Amin MA, Ahmed S, Marotte H, Rabquer BJ, et al. Expression and function of CXCL16 in a novel model of gout. Arthritis Rheum 2010;62:2536-44.

23. Koch AE, Burrows JC, Marder R, Domer PH, Leibovich SJ. Reactivity of human tissues with monoclonal antibodies to myeloid activation and differentiation antigens: an immunohistochemical study. Pathobiology 1990;58:241-8.

24. Prevoo ML, van 't Hof MA, Kuper HH, van Leeuwen MA, van de Putte LB, van Riel PL. Modified disease activity scores that include twenty-eight-joint counts: development and validation in a prospective longitudinal study of patients with rheumatoid arthritis. Arthritis Rheum 1995;38:44-8.

25. Wuyts A, Govaerts C, Struyf S, Lenaerts JP, Put W, Conings R, et al. Isolation of the CXC chemokines ENA-78, GRO $\alpha$ and GRO $\gamma$ from tumor cells and leukocytes reveals NH2-terminal heterogeneity: functional comparison of different natural isoforms. Eur J Biochem 1999;260:421-9.

26. Gerszten RE, Garcia-Zepeda EA, Lim YC, Yoshida M, Ding HA, Gimbrone MA, et al. MCP-1 and IL-8 trigger firm adhesion of monocytes to vascular endothelium under flow conditions. Nature 1999;398:718-23.

27. Proost P, Loos T, Mortier A, Schutyser E, Gouwy M, Noppen S, et al. Citrullination of CXCL8 by peptidylarginine deiminase alters receptor usage, prevents proteolysis, and dampens tissue inflammation. J Exp Med 2008;205:2085-97.

28. Loos T, Opdenakker G, Van Damme J, Proost P. Citrullination of CXCL8 increases this chemokine's ability to mobilize neutrophils into the blood circulation. Haematologica 2009;94:1346-53.

29. Loos T, Mortier A, Gouwy M, Ronsse I, Put W, Lenaerts JP, et al Citrullination of CXCL10 and CXCL11 by peptidylarginine deiminase: a naturally occurring posttranslational modification of chemokines and new dimension of immunoregulation. Blood 2008;112:2648-56.

30. Struyf S, Noppen S, Loos T, Mortier A, Gouwy M, Verbeke H, et al. Citrullination of CXCL12 differentially reduces CXCR4 and CXCR7 binding with loss of inflammatory and anti-HIV-1 activity via CXCR4. J Immunol 2009;182:666-74.

31. Chang X, Zhao Y, Sun S, Zhang Y, Zhu Y. The expression of PADI4 in synovium of rheumatoid arthritis. Rheumatol Int 2009; 29:1411-6.

32. Koch AE, Kunkel SL, Harlow LA, Johnson B, Evanoff HL, Haines GK, et al. Enhanced production of monocyte chemoattractant protein-1 in rheumatoid arthritis. J Clin Invest 1992;90:772-9.

33. Koch AE, Kunkel SL, Harlow LA, Mazarakis DD, Haines GK, Burdick MD, et al. Macrophage inflammatory protein- $1 \alpha$ : a novel chemotactic cytokine for macrophages in rheumatoid arthritis. J Clin Invest 1994;93:921-8.

34. Akahoshi T, Wada C, Endo H, Hirota K, Hosaka S, Takagishi K, et al. Expression of monocyte chemotactic and activating factor in rheumatoid arthritis: regulation of its production in synovial cells by interleukin-1 and tumor necrosis factor. Arthritis Rheum 1993;36:762-71.

35. Hatano Y, Kasama T, Iwabuchi H, Hanaoka R, Takeuchi HT, Jing $\mathrm{L}$, et al. Macrophage inflammatory protein $1 \alpha$ expression by synovial fluid neutrophils in rheumatoid arthritis. Ann Rheum Dis 1999;58:297-302.

36. Peichl P, Ceska M, Effenberger F, Haberhauer G, Broell H, 
Lindley IJ. Presence of NAP-1/IL-8 in synovial fluids indicates a possible pathogenic role in rheumatoid arthritis. Scand J Immunol 1991;34:333-9.

37. Mortier A, Gouwy M, Van Damme J, Proost P. Effect of posttranslational processing on the in vitro and in vivo activity of chemokines. Exp Cell Res 2011;317:642-54.

38. Mortier A, Loos T, Gouwy M, Ronsse I, Van Damme J, Proost P. Posttranslational modification of the NH2-terminal region of CXCL5 by proteases or peptidylarginine deiminases (PAD) differently affects its biological activity. J Biol Chem 2010;285: 29750-9.

39. Strieter RM, Polverini PJ, Kunkel SL, Arenberg DA, Burdick MD, Kasper J, et al. The functional role of the ELR motif in CXC chemokine-mediated angiogenesis. J Biol Chem 1995;270: $27348-57$.

40. Koch AE, Volin MV, Woods JM, Kunkel SL, Connors MA, Harlow LA, et al. Regulation of angiogenesis by the C-X-C chemokines interleukin- 8 and epithelial neutrophil activating peptide 78 in the rheumatoid joint. Arthritis Rheum 2001;44:31-40.

41. Holmes WE, Lee J, Kuang WJ, Rice GC, Wood WI. Structure and functional expression of a human interleukin-8 receptor. Science 1991;253:1278-80.

42. Murphy PM, Tiffany HL. Cloning of complementary DNA encoding a functional human interleukin-8 receptor. Science 1991;253: $1280-3$.

43. Persson T, Monsef N, Andersson P, Bjartell A, Malm J, Calafat J, et al. Expression of the neutrophil-activating CXC chemokine ENA-78/CXCL5 by human eosinophils. Clin Exp Allergy 2003;33: $531-7$.
44. Horuk R. Chemokine receptors. Cytokine Growth Factor Rev 2001;12:313-35.

45. Chapman RW, Phillips JE, Hipkin RW, Curran AK, Lundell D, Fine JS. CXCR2 antagonists for the treatment of pulmonary disease. Pharmacol Ther 2009;121:55-68.

46. Browning DD, Diehl WC, Hsu MH, Schraufstatter IU, Ye RD. Autocrine regulation of interleukin-8 production in human monocytes. Am J Physiol Lung Cell Mol Physiol 2000;279:L1129-36.

47. Ludwig A, Petersen F, Zahn S, Gotze O, Schroder JM, Flad HD, et al. The CXC-chemokine neutrophil-activating peptide-2 induces two distinct optima of neutrophil chemotaxis by differential interaction with interleukin-8 receptors CXCR-1 and CXCR-2. Blood 1997;90:4588-97.

48. Murphy PM, Baggiolini M, Charo IF, Hebert CA, Horuk R, Matsushima K, et al. International union of pharmacology. XXII. Nomenclature for chemokine receptors. Pharmacol Rev 2000;52: $145-76$.

49. Snir O, Rieck M, Gebe JA, Yue BB, Rawlings CA, Nepom G, et al. Identification and functional characterization of $T$ cells reactive to citrullinated vimentin in HLA-DRB1*0401-positive humanized mice and rheumatoid arthritis patients. Arthritis Rheum 2011;63: 2873-83.

50. Fan LY, He DY, Wang Q, Zong M, Zhang H, Yang L, et al. Citrullinated vimentin stimulates proliferation, pro-inflammatory cytokine secretion, and PADI4 and RANKL expression of fibroblast-like synoviocytes in rheumatoid arthritis. Scand J Rheumatol 2012;41:354-8.

51. Liddiard K, Rosas M, Davies LC, Jones SA, Taylor PR. Macrophage heterogeneity and acute inflammation. Eur J Immunol 2011;41:2503-8 